\title{
Design and Implementation of Injection Locked Oscillator Biosensors
}

\author{
M. Babay'1, C. Dalmay¹, B. Barelaud', E.C. Durmaz ${ }^{3}$, C. Baristiran Kaynak ${ }^{3}$, M. Kaynak ${ }^{3}$, D. Cordeau², A. Pothier ${ }^{1}$ \\ ${ }^{1}$ XLIM, Univ. Limoges, XLIM, UMR 7252, Limoges, France \\ ${ }^{2}$ CNRS, University of Poitiers - XLIM, UMR 7252, Angouleme, France \\ ${ }^{3}$ IHP - Leibniz-Institut für innovative Mikroelektronik, 15236 Frankfurt (Oder), Germany \\ meissa.babay@xlim.fr
}

\begin{abstract}
This paper presents the design and the implementation of a Lab-on-Chip biosensor, based on injection locked oscillators (ILO) integrated on a BiCMOS SiGe technology, dedicated to the analysis of biological cells by dielectric spectroscopy at microwave frequencies. The proposed circuit is able to evaluate the dielectric response of suspended cells circulating in a microfluidic channel. Under these conditions, a sensing accuracy of a few attoF is necessary to discriminate differences between cells, which in the case of oscillators in free running frequency would require to be able to generate variations of the oscillation frequency of the order of $10 \mathrm{kHz}$ in the vicinity of $5 \mathrm{GHz}$. With the proposed biosensor, post layout simulations show that it should be possible to measure effects of 10 attoF capacitive variation. Hence, the architecture of injection locked oscillators may prove to be the much more relevant approach at the price of a total current consumption of $37 \mathrm{~mA}$ from a $2.5 \mathrm{~V}$ supply voltage.

Keywords - RF Biosensor, BiCMOS Chip, Biological Cell Dielectric Characterization, Injection Locked Oscillators.
\end{abstract}

\section{INTRODUCTION}

In recent years, important biomedical needs have shown enormous interest in the study of biosensors that allow reliable, low cost, accurate and fast analysis of biological elements such as biomolecules or cells. Great progress has been made in the medicine and health field thanks to the development of biomedical microwave systems which bring new possibilities and perspectives in terms of diagnosis [1]. Especially, biosensors can appear very promising today to be used in the field of oncology to detect cancer cells and to help to fight cancer diseases [2]. In particular, recent works have shown the potential of IC and CMOS technology for the integration of RF characterization devices by dielectric spectroscopy [3, 4]. This sensing method consists in measuring the intracellular dielectric properties of biological samples and can, under the conditions of sufficient detection sensitivity, measure the influence of differences in physiology, pathology or morphology between different cells. The application of microwaves allows investigating the intrinsic cytoplasm since at such frequencies the electric field easily penetrates through the cell membrane. To reach the thresholds of sensitivity required, active architectures and especially those based on oscillators seem particularly attractive. Several approaches can be envisaged, whether by means of frequency counting [5], PLL (Phase Locked Loop) [6] or injection-locked oscillators [7]. In the context of biomedical sensing, the use of oscillator-based sensors demands a very strong frequency stability of the sensing oscillators enabling detecting small frequency shifts and achieving high sensitivity sensing.

In this context, this work proposes to implement a sensing system based on injection-locked oscillators with the potential to reach the high level of sensitivity required to measure dynamically the complex permittivity differences that can appear between different biological samples flowing in a microchannel filled with a liquid (cell survival medium or culture medium).

The paper is organized as follows. Section II reminds the principle of an ILO and describes the proposed circuit architecture. Section III presents the implementation and the post-layout simulation results. Finally, a conclusion is given in section IV.

\section{SYSTEM ARCHITECTURE}

This system aims to evaluate the permittivity of the biological cells passing through a microfluidic channel, by measuring the induced phase difference between two identical LC oscillators, implemented in this channel and locked on the same injection signal. At the passage of a cell in the vicinity of one of these capacitive sensors (here considered as the oscillator detector), its free-running frequency will be brought to vary with respect to the one of the second oscillator whose sensing capacitance (used here as a reference) will not be influenced or influenced differently if a different cell gets involved .

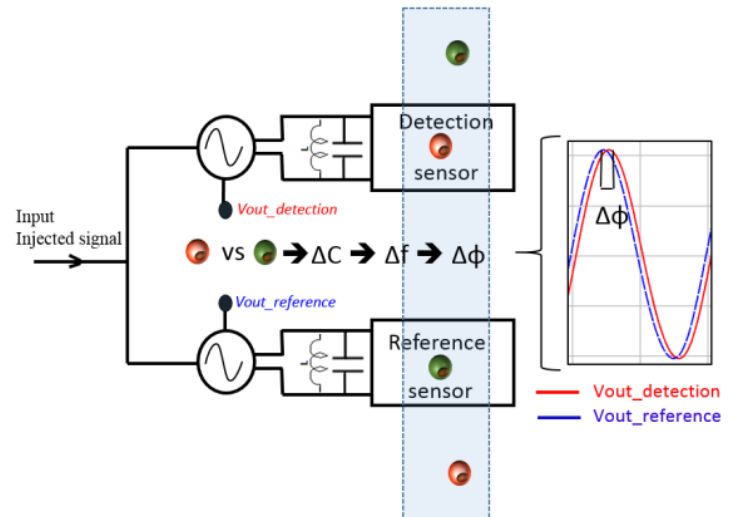

Fig.1: Schematic diagram of the sensing system 


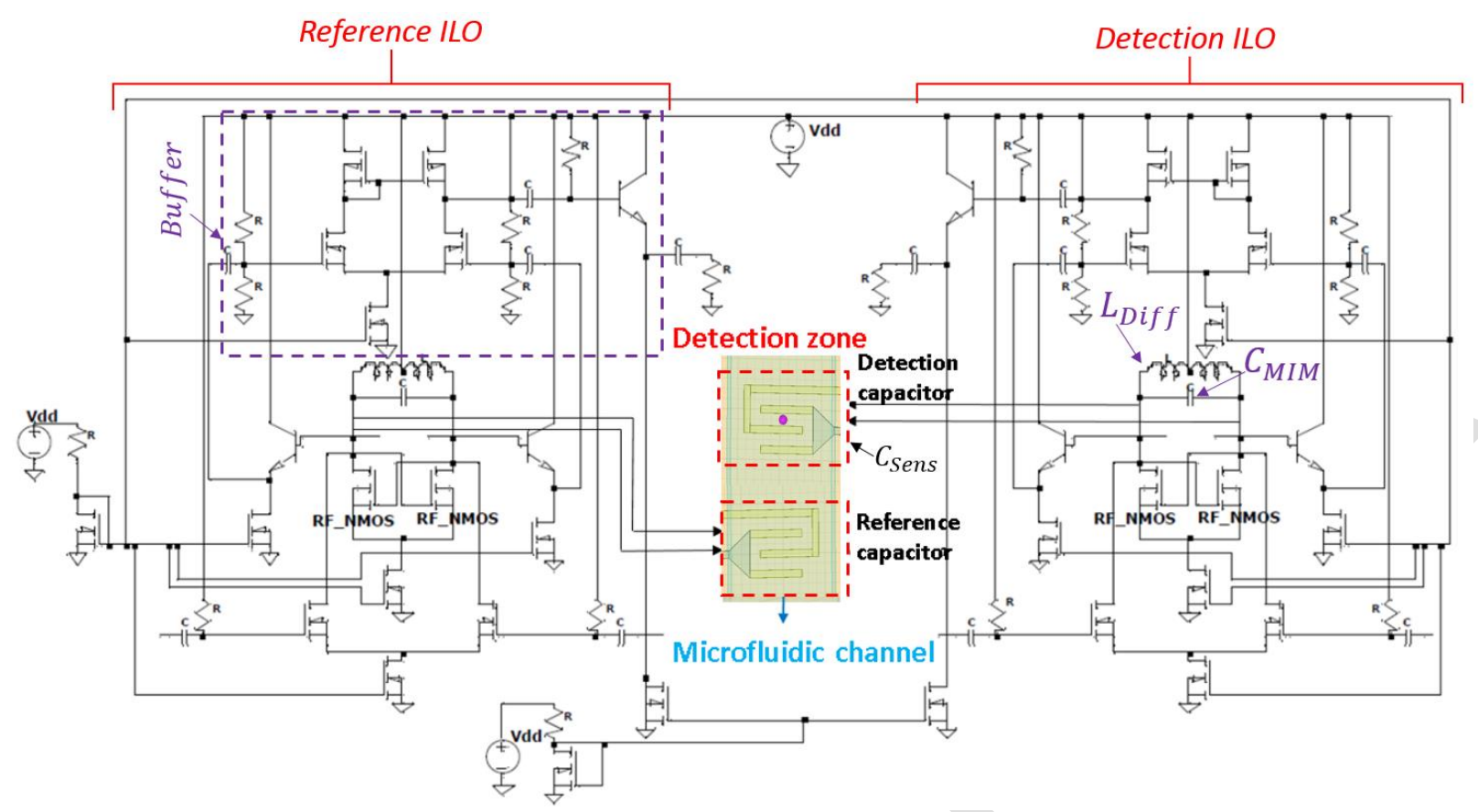

Fig. 2: Global architecture of the biosensor

As illustrated in Fig. 1, this differential measurement approach should ensure better measurement accuracy by selfcorrecting potential frequency deviations induced by the environmental perturbations.

The proposed sensing system is thus based on the use of two injection-locked oscillators. This concept was firstly introduced by Adler [8] for weak injection level, and later by Razavi [9] for high injection level. The principle of the injection is based on the fact that a master oscillator (ideally a spectrally pure source), imposes, under certain conditions, its frequency on a slave oscillator, thus generating a phase shift between the output signals of the two oscillators. This phase shift will therefore vary as a function of the free-running frequency difference between the slave and the master [8, 9]. Actually, the behavior of the injection-locked oscillator can be modeled using the Adler equation (1):

$$
\omega_{\text {det }}-\omega_{\text {inj }}=\frac{\omega_{\text {det }}}{2 Q} \cdot \frac{A_{\text {inj }}}{A_{\text {osc }}} \cdot \sin \left(\phi_{\text {det }}-\phi_{\text {inj }}\right)
$$

where $\omega_{\text {inj }}, \phi_{\text {inj }}$ and $A_{\text {inj }}$ are respectively the pulsation, the phase and the amplitude of the injection signal and $\omega_{\mathrm{det}}, \phi_{\mathrm{det}}$ and $\mathrm{A}_{\text {osc }}$ the pulsation, the phase and the amplitude of the output signal of the slave oscillator. For the targeted application, expected capacitance variation can be very low typically few hundreds to few tens of attoFarads. So in these conditions, and in order to properly detect the desired capacitive variations, it is necessary to work on low injection mode to ensure a generated phase shift that might linearly vary between $\left[-30^{\circ},+30^{\circ}\right]$.

\section{IMPLEMENTATION AND POST LAYOUT SIMULATION RESULTS}

\section{A. Circuit Design}

The proposed circuit is composed of two pair (Detection \& Reference) of differential injection locked oscillators. Each differential oscillator is based on the NMOS cross-coupled architecture as illustrated in Fig. 2. In this architecture, the RF_NMOS transistors, with a dimension equal to $\mathrm{W}=14 \mu \mathrm{m} /$ $\mathrm{L}=0.25 \mu \mathrm{m}$, provide the negative resistance to compensate the losses associated with the resonant circuit, made of a differential inductance $\left(\mathrm{L}_{\text {Diff }}\right)$, a Metal insulator Metal capacitor $\left(\mathrm{C}_{\mathrm{MIM}}\right)$ and a comb sensing capacitor $\left(\mathrm{C}_{\text {Sens }}\right)$ immersed in the cell suspension fluid; all associated in parallel. The tail current source is made using a conventional NMOS current mirror and provides the bias current. A buffer stage has been implemented, at the output of the oscillator, in order to ensure the differential to single-ended conversion and for $50 \Omega$ impedance matching.

Hence, when a cell passes in the detection zone vicinity, this may induce a small variation of the sensing capacitance of the detection oscillator's tank. As a consequence, the free running frequency of the detection oscillator should vary in similar proportions without any influence on the reference oscillator and its free running frequency. When the system is under injection, this capacitance variation results in a phase shift between both output signals of the detection oscillator and the reference oscillator. The value of this phase shift depends on the amplitude of the injected signal $A_{\text {inj }}$ (i.e. the level of injection) but also on the difference of dielectric properties between the detected cells and the support medium fluid. The considered sensing element, here, is based on a comb capacitor 
immersed at the bottom of a microfluidic channel, as shown in Fig. 2. An equivalent electrical model for this sensing capacitor has been implemented based on 3D HFSS software FEM simulations (Fig. 2.) and allows predicting accurately the effects of dielectric properties variations for different considered cells. As the detection zone is immersed in a lossy medium (microfluidic channel will be filled with an aqueous sucrose based medium of $20 \mathrm{mS} . \mathrm{m}^{-1}$ conductivity), the model considers a relatively low-quality factor equal to 15 at $5 \mathrm{GHz}$.

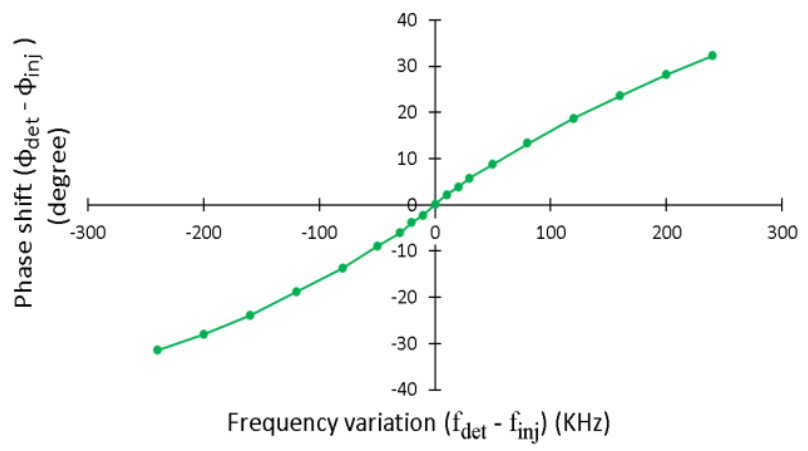

Fig.3: Single ILO expected phase variation according to its potential free running frequency change

Fig. 3 shows the impact of the variation of the free running frequency $\left(f_{\text {det }}\right)$ on the phase difference obtained between the detection oscillator output and the master signal ( $f_{\text {inj }}$ ). In Fig. 3 considering circuit simulations of the designed circuit shown on Fig.2, one can see that for an appropriate design and injection of the order of a few $\mathrm{mV}(\approx 10 \mathrm{mV})$, a frequency variation between -240 to $+240 \mathrm{kHz}$ around $5 \mathrm{GHz}$ may result in a phase shift range between $-30^{\circ}$ and $+30^{\circ}$. Now considering two consecutive cells flowing in the channel, with slightly intracellular property difference (for the present example a global relative permittivity difference of 1 ), the HFSS simulations predict a potential capacitance variation from the sensing capacitance in the order of 2 attoF. As shown in Fig. 4, the proposed circuit should be able to detect this small capacitive variation, generating a phase shift equal to $2.5^{\circ}$ when the system is injection locked. These circuit simulation results prove that the architecture of the proposed sensing system is extremely sensitive and can meet our expectations.

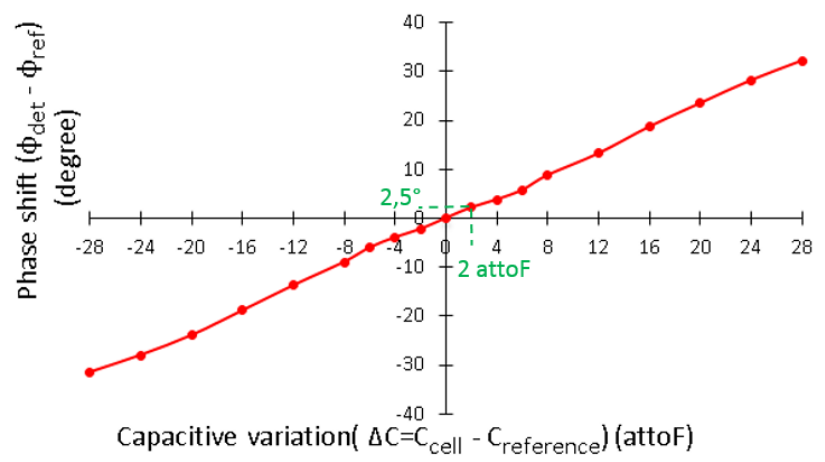

Fig.4: Simulated phase shift between the two ILOs according to the variation of the sensing capacitance.

\section{B. Implementation and post layout simulation results}

The proposed circuit was implemented in the $\mathrm{SG} 25 \mathrm{H} 4$ SiGe:C BiCMOS $0.25 \mu \mathrm{m}$ technology of IHP. The layout of the chip is shown in Fig.5. The chip dimension is $2 * 7 \mathrm{~mm}^{2}$ including all the pads. Extra lateral space is here occupied by $2.5 \mathrm{~mm}$ long microstrip RF lines allowing bringing RF signals to the chip core and passing under a PDMS (polydimethylsiloxane) cover used to form the microfluidic channel.

The extraction procedure performed with Assura tool is used to estimate layout parasitic components. By considering all the parasitic elements generated by the layout, post layout simulations, performed with Cadence Spectre RF, show that the free-running frequency of the LC oscillator should be $4.96 \mathrm{GHz}$ when no injection is applied.

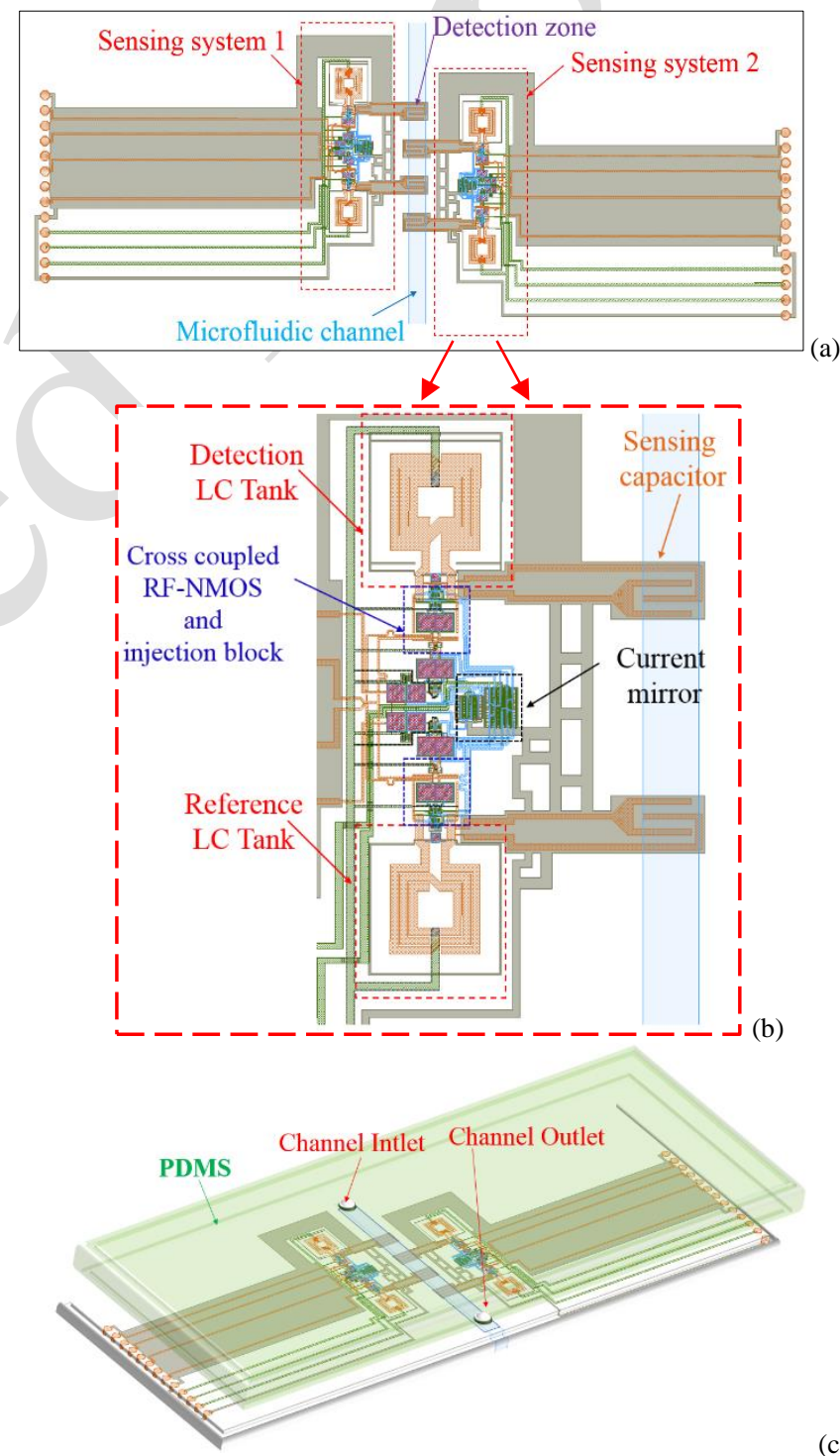

(c)

Fig.5: Chip micrograph showing 2 ILO sensors with their reference implemented in series on the same microfluidic chip (a), Layout of the sensing system (b), Chip with PDMS packaging (c) 
As shown in Fig. 5(a), the chip contains two identical sensing systems $(1 \& 2)$ implemented face to face regarding the central microfluidic channel to allow a double check of the measured dielectric property for each cell flowing in the chip. Fig. 5(b) details the implemented sensing architecture, where the detection $\&$ reference oscillators can operate in free running oscillation mode or in injection locked mode (preferred for high sensitivity sensing) using for the injected signal an external very low phase noise $\mathrm{CW}$ source (commercial microwave signal generator). Injected signal can be applied to the chip thanks to the low loss 50ohm microstrip lines. Fig. 5 (c) illustrates how the chip should be packaged using a $6 \mathrm{~mm}$ wide embossed PDMS cover to close the microfluidic channel, pre-etched in the top BEOL CMOS layer stack.

As shown in the post-layout simulations presented in Fig.6, considering a $50 \mathrm{mV}$ magnitude injection signal $\left(A_{i n j}\right)$, set at $4.96 \mathrm{GHz}$, the two detection and reference oscillators became locked and generate a phase shift $\phi_{d e t}-\phi_{r e f}=0^{\circ}$ when no cell passed above their respective sensing capacitors.

Considering now, the case of a single cell passing in the vicinity of the sensing capacitor that induces a 500attoF capacitance change regarding the reference capacitor, post layout simulation predicts a resulting phase shift between both detector \& reference ILO output signals equal to $12.6^{\circ}$, as shown in Fig. 7.

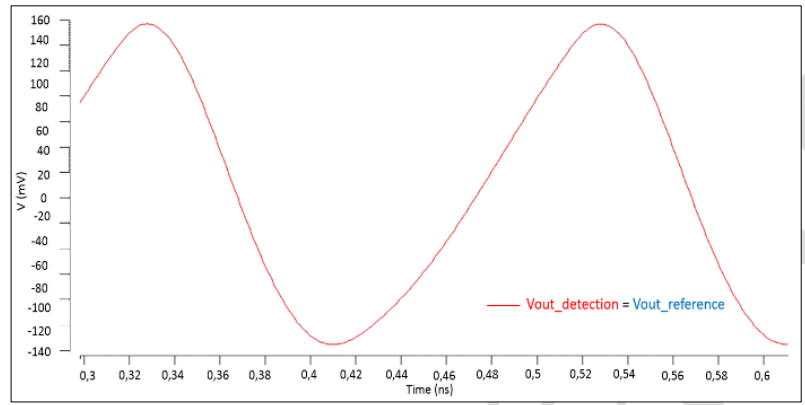

Fig.6: Output voltage of the detection system when $f_{\text {detc }}=f_{\text {inj }}$

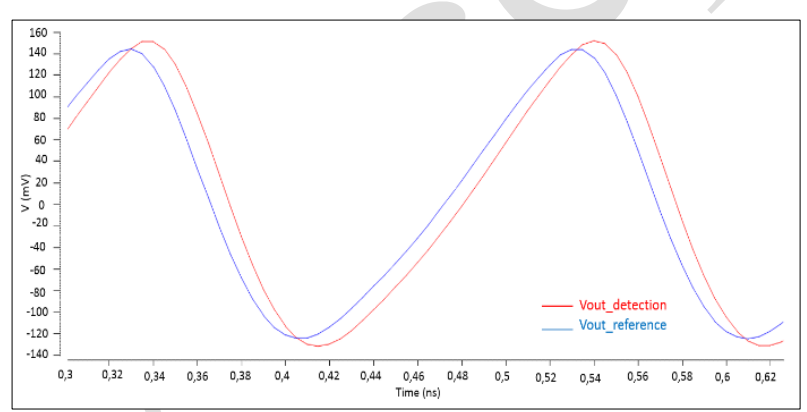

Fig.7: Output voltage of the sensing system when a cell passes through the detection zone (i.e $\mathrm{C}_{\text {Cell }}-\mathrm{C}_{\mathrm{Ref}}=500 \mathrm{attoF}$ )

Simulation parameters and expected performance of the proposed sensing system are summarized in Table I. As one can see, the proposed sensing system may be able to detect at least 10 attoF capacitance change and probably even less, that should allow reaching a satisfactory measurement resolution to monitor differences of intracellular dielectric properties between cells.
Table I: Post layout simulated performance

\begin{tabular}{|c|c|c|c|c|c|c|c|}
\hline $\begin{array}{c}\text { Capacitive } \\
\text { variation }\end{array}$ & \multicolumn{2}{|c|}{10 attoF } & $\begin{array}{c}20 \\
\text { attoF }\end{array}$ & $\begin{array}{c}100 \\
\text { attoF }\end{array}$ & $\begin{array}{c}500 \\
\text { attoF }\end{array}$ & $1 \mathrm{fF}$ & $2 \mathrm{fF}$ \\
\hline $\begin{array}{c}\text { Injected } \\
\text { input } \\
\text { voltage } \\
(\mathrm{mV})\end{array}$ & 10 & 20 & 20 & 50 & 50 & 100 & 100 \\
\hline $\begin{array}{c}\text { Simulated } \\
\text { phase } \\
\text { shift }\left(^{\circ}\right)\end{array}$ & 11.9 & 8 & 15.5 & 3.6 & 12.6 & 13.9 & 25.7 \\
\hline
\end{tabular}

\section{CONCLUSION}

A high sensitivity sensing system based on two injection locked oscillators operating at $5 \mathrm{GHz}$ and integrated in a BiCMOS SiGe: C $0.25 \mu \mathrm{m}$ process is presented. Post layout Cadence Spectre RF simulations, considering all the parasitic elements generated by the layout, show that such device should well detect the passing of biological cells in a microfluidic channel and might be able to distinguish dielectric properties differences between cells. Chips are currently under fabrication process, with first chip tests scheduled before summer allowing us to present preliminary performances during the conference.

\section{ACKNOWLEDGMENT}

This project has received funding from the European Union's Horizon 2020 research and innovation programme under grant agreement No 737164.

\section{REFERENCES}

[1] K. Grenier et al., "Recent Advances in Microwave-Based Dielectric Spectroscopy at the Cellular Level for Cancer Investigations", IEEE Transaction on MTT, Vol. 61, no. 5, pp. 2023-2030, May 2013

[2] L.Y Zhang et al., "Discrimination of Colorectal Cancer Cell Lines using Microwave Biosensors", Sensors and Actuators A: physical, Vol. 216, pp. 405-416, 2014.

[3] M. Kaynak et al, "BiCMOS integrated microfluidic platform for BioMEMS applications", IEEE IMS 2014.

[4] A.Manickam et al, "A CMOS Electrochemical Impedance Spectroscopy Biosensor Array for Label-Free Biomolecular Detection", IEEE International Solid-State Circuits Conference, 2010.

Hua Wang et al, "Phase Noise and Fundamental Sensitivity of OscillatorBased Reactance Sensors", IEEE Transactions on MTT, vol. 61, no. 5, May 2013

[5] Yunseog Hong et al, "A Label-Free Biosensing Platform Using a PLL", IEEE Transactions on Biomedical Circuits and Systems, vol. 9, no. 3, Jun 2015.

[6] J.-C. Chien et al, "Oscillator-Based Reactance Sensors With Injection Locking for High-Throughput Flow Cytometry using microwave dielectric spectroscopy", IEEE Journal of Solid-State Circuits, vol. 51, no. 2, Feb 2016

[7] R. Adler et al, "A study of locking phenomena in oscillators," Proc. IRE, vol. 34, pp. 351-357, Jun. 1946

[8] B. Razavi et al, "A study of injection locking and pulling in oscillators," IEEE J. Solid-State Circuits, vol. 39, no. 9, pp. 1415-1424, Sep. 2004. 